GLASNIK MATEMATIČKI

Vol. 40(60)(2005), $323-331$

\title{
EQUIVARIANT FIBRANT SPACES
}

\author{
Alexander Bykov and Marcelino Texis \\ Benemérita Universidad Autónoma de Puebla, México
}

Abstract. In this paper the concept of a $G$-fibrant space is introduced. It is shown that any compact metrizable group $G$ is a $G$-fibrant.

\section{INTRODUCTION}

The general approach to the concept of a fibrant object is the following (c.f.[5]): if in a category $\mathcal{C}$ some class $\Sigma$ of morphisms is specified then an object $Y$ of $\mathcal{C}$ is called $\Sigma$-fibrant if for every morphism $s \in \Sigma, s: A \rightarrow X$, and every morphism $f: A \rightarrow Y$ there is a morphism $F: X \rightarrow Y$ such that $F \circ s=f$. The classical fibrant objects appear in [9] for the closed model categories where $\Sigma$ is the class of trivial cofibrations. A fibrant space in the sense of F. Cathey is a $\Sigma$-fibrant object, where $\Sigma$ is the class of $S S D R$-maps in the category of metrizable spaces ([6]). In the present paper we provide an equivariant version of a fibrant space.

It is well-known (see [8]) that every compact metrizable group can be represented as an inverse limit of a sequence of Lie groups bonded by fibrations (Proposition 3.3), and therefore it is already a fibrant space in the sense of F. Cathey. On the other hand, due to R. Palais ([7]), every compact Lie group $G$ is a $G$-ANR (Proposition 3.2) and hence it is a $G$-fibrant space. These are the basic facts utilized in the proof of our main theorem (Theorem 3.1 ): every compact metrizable group $G$ is a $G$-fibrant space. This result justifies the consideration of equivariant fibrant spaces. Also it is clear that equivariant fibrant spaces as well as equivariant $S S D R$-maps can be used in the construction of the equivariant strong shape category following the way of F. Cathey, which is given in [6] for the "non-equivariant" case.

2000 Mathematics Subject Classification. 54C55, 54C56, 54H15, 54B15.

Key words and phrases. Fibration, fibrant space, G-ANR.

Supported in part by CONACYT Grant 32132-E. 


\section{THE BASIC NOTIONS}

The basic definitions and facts of the equivariant theory, that is the theory of $G$-spaces and $G$-maps, can be found in [4]. Throughout the paper the letter $G$ will denote a compact Hausdorff group. By $G-A(N) R$, it is denoted the class of $G$-equivariant absolute (neighborhood) retracts for all $G$-metrizable spaces (see, for instance, [2] for the equivariant theory of retracts). In this paper all $G$-spaces are assumed to be metrizable.

A closed invariant subspace $A$ of a $G$-space $X$ is called a $G$-shape strong deformation retract of $X$ if there exists a $G$-equivariant embedding $i: X \hookrightarrow Y$ for some $G$-AR space $Y$ such that for any pair of invariant neighborhoods $U$ and $V$ of $i(X)$ and $i(A)$ respectively in $Y$, there is a $G$-homotopy $H: X \times I \rightarrow$ $U$ rel. $A$ such that $H(x, 0)=i(x)$ and $H(x, 1) \in V$ for any $x \in X$.

Note that if for a $G$-pair $(X, A)$ an embedding $i: X \hookrightarrow M$ satisfies the conditions of the above definition then these conditions hold for any other closed $G$-equivariant embedding $j: X \hookrightarrow Z$ where $Z$ is a $G$-AR space.

A closed $G$-equivariant embedding $s: A \hookrightarrow X$ is called $a G$-SSDR-map if $s$ embeds $A$ in $X$ as a $G$-shape strong deformation retract of $X$.

A $G$-space $Y$ is called $a G$-fibrant if for every $G$-SSDR-map $s: A \hookrightarrow X$ and every $G$-map $f: A \rightarrow Y$, there exists a $G$-map $F: X \rightarrow Y$ such that $F \circ s=f$.

Recall that a map $p: E \rightarrow B$ is a $G$-fibration if for every $G$-space $X$ and every commutative diagram of $G$-maps

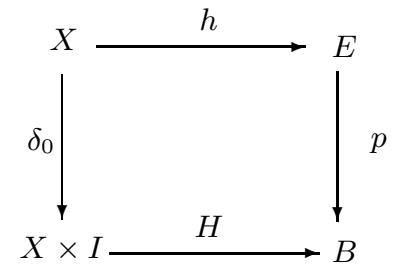

where $\delta_{0}(x)=(x, 0)$, there exists $\widetilde{H}: X \times I \rightarrow E$ such that $\widetilde{H} \circ \delta_{0}=h$ and $p \circ \widetilde{H}=H$.

For example, the $G$-fibrations naturally appear in the following situation. Let $U$ be a $G$-space. The space $U^{I}$ of paths $\omega: I \rightarrow U$, provided with the compact-open topology, can be treated as a $G$-space with the action: $(g \star \omega)(t)=g \omega(t)$. Then the projection $p: U^{I} \rightarrow U \times U$, given by $p(\omega)=$ $(\omega(0), \omega(1))$, is a $G$-fibration.

The following theorem is an equivariant version of Theorem 1.2 of [6].

Theorem 2.1. Let $s: A \hookrightarrow X$ be a closed $G$-embedding. Then the following conditions are equivalent:

(a) s is a G-SSDR-map; 
(b) for any G-equivariant map $f: A \rightarrow Y$, where $Y$ is $G$-ANR, there is a G-equivariant extension $\widetilde{f}: X \rightarrow Y$ such that $\tilde{f} \circ s=f$, and if $\widetilde{f}_{1}, \widetilde{f}_{2}: X \rightarrow Y$ are any two such extensions, then $\tilde{f}_{1} \simeq_{G} \widetilde{f}_{2}$ rel. $s(A)$;

(c) For any $G$-fibration $p: E \rightarrow B$, where $E$ and $B$ are $G$-ANR-spaces and any commutative diagram of $G$-equivariant maps

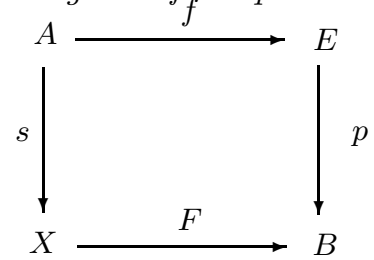

there exists a G-equivariant map $\widetilde{F}: X \rightarrow E$ such that $\widetilde{F} \circ s=f$ and $p \circ \widetilde{F}=F$.

We shall give the proof of the theorem though it is quite analogous to the proof of its "non-equivariant" case.

Proof. (a) $\Rightarrow$ (b). Clearly, we can assume that $A \subset X$ and $s(a)=a$. Let $X \hookrightarrow M$ be an equivariant closed embedding of $X$ in some $G$-AR space $M$ (See [3], Proposition 1). Since each $G$-AR space for metrizable spaces is a $G$-AE ([3], Proposition 2), there is a $G$-extension $\widehat{f}: V \rightarrow Y$ of $f$ on some invariant open neighborhood $V$ of $A$ in $M$. By the definition of a $G-S S D R$ map, we can find a $G$-homotopy $H: X \times I \rightarrow M$ such that $H(x, 0)=x$, $H(x, 1) \in V$ and $H(a, t)=a$ for $x \in X, a \in A, t \in I$. The required extension $\widetilde{f}: X \rightarrow Y$ can be given by $\widetilde{f}(x)=\widehat{f}(H(x, 1))$.

Let $\widetilde{f}_{1}, \widetilde{f}_{2}: X \rightarrow Y$ be two $G$-extensions of $f$. Define a $G$-equivariant map $F: X \times 0 \cup A \times I \cup X \times 1 \rightarrow Y$ by $F(x, 0)=\widetilde{f}_{1}(x), F(x, 1)=\widetilde{f}_{2}(x)$, $F(a, t)=f(a)$ for $x \in X, a \in A, t \in I$. Considering $X$ as a closed invariant subset of a $G$-AR space $M$, and therefore $X \times I$ as a closed invariant subset of the $G$-AR space $M \times I$, we find a $G$-extension $\bar{F}: W \rightarrow Y$ of $F$ on some invariant neighborhood $W$ of $X \times 0 \cup A \times I \cup X \times 1$ in $M \times I$. Clearly, one can choose an invariant neighborhood $U$ of $X$ in $M$ such that $U \times\{0\} \subset W$ and $U \times\{0\} \subset W$. Besides, a standard compactness argument guarantees the existence of an invariant neighborhood $V$ of $A$ in $M$ such that $V \times I \subset W$. Taking a $G$-homotopy $D: X \times I \rightarrow U$ such that $D(x, 0)=x, D(x, 1) \in V$ and $D(a, t)=a$ for $x \in X, a \in A, t \in I$, we can establish $G$-homotopies $F^{\prime}: \widetilde{f}_{1} \simeq_{G} h_{1}$ rel. $A, F^{\prime \prime}: \widetilde{f}_{2} \simeq_{G} h_{2}$ rel. $A$ and $H: h_{1} \simeq_{G} h_{2}$ rel. $A$ by $F^{\prime}(x, t)=\bar{F}(D(x, t), 0), F^{\prime \prime}(x, t)=\bar{F}(D(x, t), 1)$ and $H(x, t)=\bar{F}(D(x, 1), t)$. Thus $\widetilde{f}_{1} \simeq_{G} \widetilde{f}_{2}$ rel. $A$.

(b) $\Rightarrow($ c). Since $E$ is a $G$-ANR there exists a $G$-extension $\bar{F}: X \rightarrow E$ such that $\bar{F} \circ s=f$. We have $F \circ s=p \circ \bar{F} \circ s$ and by the second part of (b) there is a $G$-homotopy $H: F \simeq_{G} p \bar{F}$ rel. $s(A)$. Applying the covering 
homotopy property we get a $G$-homotopy $\widetilde{H}: X \times I \rightarrow E, \widetilde{H}: \tilde{F} \simeq_{G} \widetilde{F}$ rel. $s(A)$, such that $p \circ \widetilde{H}=H$. So $\widetilde{F} \circ s=f$ and $p \circ \widetilde{F}=F$ as required.

(c) $\Rightarrow$ (a). As above, we can assume that $X$ is an invariant closed subset of some $G$-AR space $M$ and that $A$ is an invariant closed subset of of $X$, so $s(a)=a$ for $a \in A$. Let $U$ and $V$ be invariant open neighborhoods of $X$ and $A$ respectively in $M$. First applying (c) to the $G$-fibration $V \rightarrow *$ and the inclusion $i: A \rightarrow V$ we get a $G$-map $r: X \rightarrow V$ such that $r \circ s=i$. Afterwards applying (c) to the commutative diagram

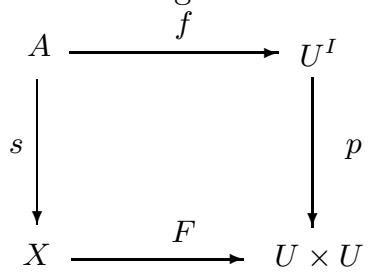

where $p(\omega)=(\omega(0), \omega(1)), f(a)(t)=a, F(x)=(x, r(x))$, we obtain a $G$-map $\bar{F}: X \rightarrow U^{I}$ such that $\bar{F} \circ s=f, p \circ \bar{F}=F$. Now observe that the map $D: X \times I \rightarrow U$ defined by $D(x, t)=\bar{F}(x)(t)$ satisfies the conditions of the definition of a $G$-SSDR-map.

Corollary 2.2. Every $G$-ANR is a $G$-fibrant space.

\section{MAIN RESUlt}

The main result of this paper is the following

ThEOREM 3.1. Every compact metrizable group $G$ is a $G$-fibrant space.

In the proof of this theorem, we shall use the propositions given below.

Proposition 3.2. ([7], Proposition 1.6.6) Let $G$ be a compact Lie group and $H$ be its closed subgroup. Then $G / H$ is a $G$-ANR space.

The following result is actually proved in the classical book of Pontrjagin [8]. Note that it can be easily obtained from Corollary 4.4 of [4]: for every neighborhood $U$ of the unit $e$ of a compact group $G$, there exists a group morphism $\varphi: G \rightarrow \mathbf{O}(n)$ such that $\operatorname{ker} \varphi \subseteq U$.

Proposition 3.3. Let $G$ be a compact metrizable group. Then there exists a decreasing sequence $\left\{N_{i}\right\}_{i \in \mathbb{N}}$ of its normal closed subgroups such that the quotient groups $G / N_{i}$ are Lie groups, $\bigcap_{i \in \mathbb{N}} N_{i}=\{e\}$ and

$$
\underset{\lim }{\longleftarrow}\left\{G / N_{i}, q_{i}^{j}\right\}=G
$$

where $q_{i}^{j}: G / N_{j} \rightarrow G / N_{i}, j \geq i$, are the natural projections.

We omit a routine proof of the following statement. 
Proposition 3.4. Let $G$ be a compact metrizable group and $\left\{N_{i}\right\}_{i \in \mathbb{N}}$ be a sequence of its closed normal subgroups satisfying Proposition 3.3.

(a) If $X$ is a $G$-space, then

$$
X=\lim _{\longleftarrow}\left\{X / N_{i}, p_{i}^{j}\right\}
$$

where $p_{i}^{j}: X / N_{j} \rightarrow X / N_{i}, j \geq i$, are the natural projections.

(b) Let $X$ and $Y$ be $G$-spaces represented according to (a) as

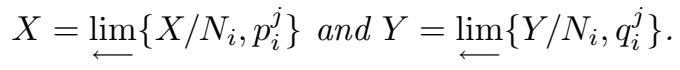

If the $G / N_{i}$-maps $f_{i}: X / N_{i} \rightarrow Y / N_{i}, i \in \mathbb{N}$, are such that $q_{i}^{i+1} f_{i+1}=$ $f_{i} p_{i}^{i+1}$, i.e. the diagram

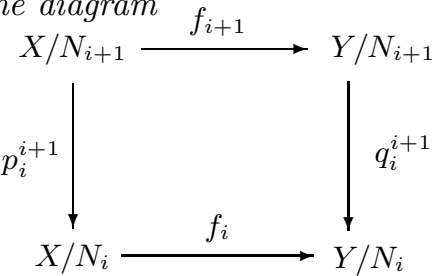

commutes for each $i \in \mathbb{N}$, then there exists a unique $G$-map $f: X \rightarrow Y$ such that $q_{i} f=f_{i} p_{i}$ for each $i$, where $p_{i}: X \rightarrow X / N_{i}, q_{i}: Y \rightarrow Y / N_{i}$ are $N_{i}$-orbit projections.

Proposition 3.5. Let $G$ be a compact group and $N$ be a closed normal subgroup of $G$. If $s: A \rightarrow X$ is a G-SSDR-map, then the induced map $s / N: A / N \rightarrow X / N$ is a $G / N-S S D R$-map.

Proof. Let $j_{0}: X / N \hookrightarrow Y$ be a closed $G / N$-embedding of $X / N$ in a $G / N-A N R$ space $Y$. By Lemma 1 of [1] there exist a $G$-space $Z$ and a closed $G$-embedding $\widehat{j}_{0}: X \hookrightarrow Z$ such that $Z / N=Y$ and $j_{0} \circ p=q_{1} \circ \widehat{j}_{0}$, where $p: X \rightarrow X / N, q_{1}: Z \rightarrow Y$ are the $N$-orbit maps. Let $\widehat{j}_{1}: Z \hookrightarrow M$ be a closed $G$-embedding of $Z$ in a $G$ - $A R$ space $M$ (See [3], Proposition 1). Then by Theorem 1 of [2] $M / N$ is a $G / N-A N R$ space and we get a closed $G / N$-embedding $j=j_{1} \circ j_{0}: X / N \hookrightarrow M / N$, where the embedding $j_{1}: Z / N \hookrightarrow M / N$ is induced by $\widehat{j}_{1}$. Moreover, for the closed $G$-embedding $\widehat{j}=\widehat{j}_{1} \circ \widehat{j}_{0}: X \hookrightarrow M$ and the $N$-orbit map $q: M \rightarrow M / N$ we have $q \circ \widehat{j}=j \circ p$.

Now let $U$ and $V$ be invariant neighborhoods of $X / N$ and $A / N$ respectively in $M / N$. Then $\widehat{U}=q^{-1}(U)$ and $\widehat{V}=q^{-1}(V)$ are invariant neighborhoods of $X$ and $A$ respectively in $M$. Since $s: A \hookrightarrow X$ is a $G$-SSDR-map there exists a $G$-homotopy $\widehat{H}: X \times I \rightarrow \widehat{U}$ rel. $A$ such that $\widehat{H}(x, 0)=x$ and $\widehat{H}(x, 1) \in \widehat{V}$. It is clear that the induced $G / N$-homotopy $H: X / N \times I \rightarrow U$, defined by $H(N(x), t)=N(\widehat{H}(x, t))$, satisfies the analogous properties and it means that $s / N: A / N \hookrightarrow X / N$ is a $G / N-S S D R$-map.

We need the following version of the covering homotopy theorem (compare [4], Ch. II, Theorem 7.3). 
Proposition 3.6. Let $G$ be a compact Lie group and $N$ be a normal closed subgroup of $G$. Suppose that for a $G$-space $X, a G / N$-homotopy $H$ : $X / N \times I \rightarrow G / N$ and $a G$-map $h: X \rightarrow G$ are such that the following diagram commutes

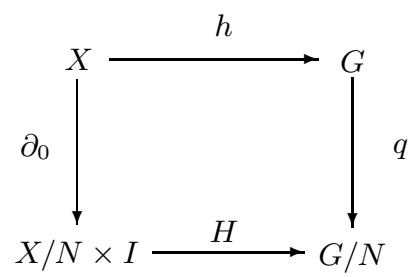

where $\partial_{0}(x)=(p(x), 0)$ and $p: X \rightarrow X / N, q: G \rightarrow G / N$ are the $N$ orbit maps. Then there exists a G-homotopy $\widetilde{H}: X \times I \rightarrow G$ such that $\widetilde{H}(x, 0)=h(x)$ and $q \circ \widetilde{H}=H \circ\left(p \times 1_{I}\right)$.

Moreover, if $A$ is an invariant closed subset of $X$ such that $H(p(a), t)=$ $H(p(a), 0)$ for any $a \in A, t \in I$, then the covering homotopy $\widetilde{H}$ can be chosen so that $\widetilde{H}(a, t)=h(a)$ for any $a \in A, t \in I$.

Proof. Note that the existence of the $G$-map $h: X \rightarrow G$ implies that the action of the group $G$ on $X$ is of a quite simple structure. Indeed, let $S=h^{-1}(e)$, where $e$ is the unit element of the group $G$, and let $\rho: X \rightarrow S$ be a map defined by $\rho(x)=(h(x))^{-1} x$. Then $\rho$ is a retraction such that $\rho(g x)=\rho(x)$ for any $g \in G, x \in X$. Now consider the product $G \times S$ as a $G$-space endowed with the action $g\left(g^{\prime}, s\right)=\left(g g^{\prime}, s\right)$. It can be easily verified that the map $\varphi: X \rightarrow G \times S$, given by $\varphi(x)=(h(x), \rho(x))$, is a $G$-map and, moreover, it is a $G$-equivalence because the $G$-map $\psi: G \times S \rightarrow X$, where $\psi(g, s)=g s$, is the inverse map for $\varphi$. In fact, we shall use this $G$-equivalence in the construction of the covering homotopy $\widetilde{H}$.

Let $F: S \times I \rightarrow G / N$ be a homotopy defined by $F(s, t)=H(p(s), t)$. For the given invariant closed subset $A \subseteq X$, let $U=A \cap S$, that is $U=\rho(A)$. Then, if $u \in U$, we have $F(u, t)=H(p(u), t)=H(p(u), 0)=F(u, 0)$ for any $t \in I$.

Since $N$ is a Lie group, the projection $q: G \rightarrow G / N$ is a locally trivial fibration (see [4], Ch. II, Theorem 5.8) and hence it has the regular homotopy lifting property. In particular, considering the commutative diagram

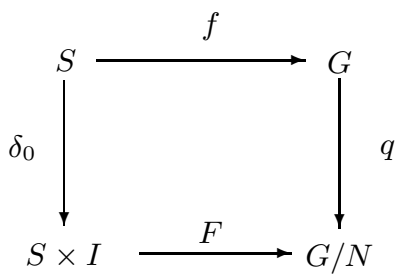


where $f(s)=e$ and $\delta_{0}(s)=(s, 0)$ for $s \in S$, one can find a homotopy $\widetilde{F}$ : $S \times I \rightarrow G$ which preserves the commutativity of the diagram, that is to say, $q \circ \widetilde{F}=F, \widetilde{F} \circ \delta_{0}=f$ and, moreover, $\widetilde{F}(u, t)=e$ for any $u \in U, t \in I$.

Finally, the required covering homotopy $\widetilde{H}: X \times I \rightarrow G$ can be defined by $\widetilde{H}(x, t)=h(x) \widetilde{F}(\rho(x), t)$. The verification of this fact is straightforward.

Indeed, we have $\widetilde{H}(x, 0)=h(x) \widetilde{F}(\rho(x), 0)$, but

$$
\widetilde{F}(\rho(x), 0)=\widetilde{F} \circ \delta_{0}(\rho(x))=f(\rho(x))=e,
$$

and hence $\widetilde{H}(x, 0)=h(x)$.

Since $q: G \rightarrow G / N$ is a group morphism and $q \circ \widetilde{F}=F$, we get

$$
q(\widetilde{H}(x, t))=q(h(x)) q(\widetilde{F}(\rho(x), t))=q(h(x)) F(\rho(x), t) .
$$

By the definition of the homotopy $F$, we have $F(\rho(x), t)=H(p(\rho(x)), t)$, but $H$ is a $G / N$-map, and therefore, $q(h(x)) H(p(\rho(x)), t)=H(q(h(x)) p(\rho(x)), t)$. Reminding that $q: G \rightarrow G / N$ and $p: X \rightarrow X / N$ are $N$-orbit maps, we obtain

$$
q(h(x)) p(\rho(x))=p(h(x) \rho(x))=p(x) .
$$

Thus $q(\widetilde{H}(x, t))=H(p(x), t)$, that is $q \circ \widetilde{H}=H \circ\left(p \times 1_{I}\right)$.

Besides, if $a \in A$, we have $\rho(a) \in U$, and therefore,

$\widetilde{H}(a, t)=h(a) \widetilde{F}(\rho(a), t)=h(a) e=h(a)$ for any $t \in I$.

Proof of Theorem 3.1. Let $j: A \hookrightarrow X$ be a $G$-SSDR-map and $f$ : $A \rightarrow G$ be a $G$-map. In order to show that $G$ is a $G$-fibrant, we must find a $G$-map $F: X \rightarrow G$ such that $F \circ j=f$.

According to Proposition 3.3, we represent the group $G$ as an inverse limit of Lie groups $G=\lim \left\{G / N_{i}, q_{i}^{j}\right\}$.

The $G$-maps $j$ and $f$ induce for each $k$ the $G / N_{k}$-maps $j_{k}=j / N_{k}$ : $A / N_{k} \hookrightarrow X / N_{k}, f_{k}=f / N_{k}: A / N_{k} \rightarrow G / N_{k}$. Then for each $k$ the following diagram commutes

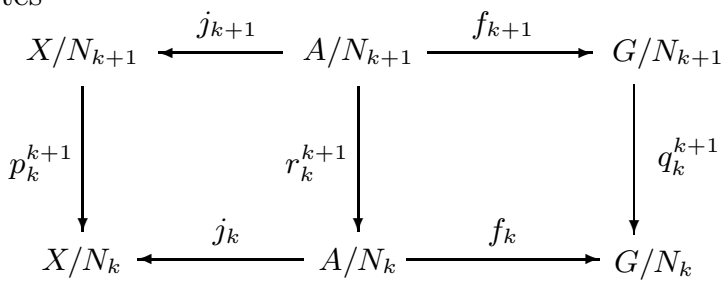

where $p_{k}^{k+1}, q_{k}^{k+1}, r_{k}^{k+1}$ are the natural projections. It is clear that these projections can be treated as orbit projections with respect to the action of the closed subgroup $N_{i} / N_{i+1}$ of the Lie group $G / N_{i+1}$ on $X / N_{i+1}, A / N_{i+1}$ and $G / N_{i+1}$ respectively. For each $k$ the map $j_{k}$ is a $G / N_{k}-S S D R$-map by Proposition 3.5, and $G / N_{k}$ is a $G / N_{k}$-ANR by Proposition 3.2, and therefore there exists a $G / N_{k}$-equivariant extension $F_{k}: X / N_{k} \rightarrow G / N_{k}$ of $f_{k}$ such that 
$F_{k} j_{k}=f_{k}$. Using these extensions we shall construct by induction $G / N_{k^{-}}$ maps $T_{k}: X / N_{k} \rightarrow G / N_{k}$ satisfying the conditions $T_{k} p_{k}^{k+1}=q_{k}^{k+1} T_{k+1}$ and $T_{k} j_{k}=f_{k}$ for every $k \in \mathbb{N}$. Let $T_{1}=F_{1}$ and suppose that $T_{k}$ is already found. We have to construct the map $T_{k+1}$.

One has

$$
T_{k} j_{k} r_{k}^{k+1}=f_{k} r_{k}^{k+1}=q_{k}^{k+1} f_{k+1}=q_{k}^{k+1} F_{k+1} j_{k+1}=F_{k}^{*} p_{k}^{k+1} j_{k+1}=F_{k}^{*} j_{k} r_{k}^{k+1},
$$

where $F_{k}^{*}: X / N_{k} \rightarrow G / N_{k}$ is the $G / N_{k}$-map induced by $F_{k+1}$. Hence $T_{k} j_{k}=$ $F_{k}^{*} j_{k}$ because $r_{k}^{k+1}$ is surjective. According to Theorem 2.1, there is a $G / N_{k^{-}}$ homotopy $H: F_{k}^{*} \simeq T_{k}$ rel. $j_{k}\left(A / N_{k}\right), H: X / N_{k} \times I \rightarrow G / N_{k}$.

Now consider the following commutative diagram

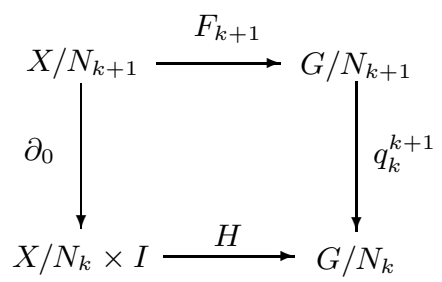

where $\partial_{0}([x])=\left(p_{k}^{k+1}[x], 0\right)$ for $[x]=N_{k+1}(x) \in X / N_{k+1}$.

Taking into account this diagram, we are going to apply Proposition 3.6 to the Lie group $G / N_{k+1}$ acting on $X / N_{k+1}$ and to its closed normal subgroup $N_{k} / N_{k+1}$. Note that one can consider the $G / N_{k+1}$-space $X / N_{k}$ as the orbit space $X / N_{k+1} / N_{k} / N_{k+1}$.

By Proposition 3.6, we get a $G / N_{k+1}$-homotopy

$$
\widetilde{H}: X / N_{k+1} \times I \rightarrow G / N_{k+1}
$$

such that $\widetilde{H}([x], 0)=F_{k+1}([x]), q_{k}^{k+1}\left(\widetilde{H}([x], t)=H\left(p_{k}^{k+1}([x]), t\right)\right.$ and, for any $t \in I, \widetilde{H}([a], t)=F_{k+1}([a])$ if $[a] \in j_{k+1}\left(A / N_{k+1}\right)$. Putting $T_{k+1}([x])=$ $\widetilde{H}([x], 1)$ we get the required $G / N_{k+1}$-map $T_{k+1}: X / N_{k+1} \rightarrow G / N_{k+1}$. This completes the inductive step.

The sequence $\left\{T_{k}\right\}_{k \in \mathbb{N}}$, according to Proposition 3.4, determines a unique $G$-map $F: X \rightarrow G$ such that $q_{k} F=T_{k} p_{k}$ for each $k$. Since $T_{k} j_{k}=f_{k}$ for each $k$, we can state that $F \circ j=f$.

ACKNOWLEDGEMENTs.

The authors wish to thank the referee for many helpful comments and suggestions.

\section{REFERENCES}

[1] S.A. Antonyan, Preservation of $k$-connectedness by a symmetric $n$-th power functor, Moscow Univ.Math. Bull. 49 (1994) 22-25. 
[2] S.A. Antonyan, Extensorial properties of orbit spaces of proper group actions, Topology and Appl. 98 (1999) 35-46.

[3] S.A. Antonyan, S.Mardešić, Equivariant Shape, Fund. Math. 127 (1987), 213-224.

[4] G.E.Bredon, Introduction to Compact Transformation Groups, Academic Press, New York, 1972.

[5] A. I. Bykov and L. G. Zerkalov, Cotelescopes and approximate lifting properties in shape theory, Topology and Appl. 73 (1996), 197-212.

[6] F. Cathey, Strong shape theory, in: Shape Theory and Geometric Topology, Lecture Notes in Math. 870, Springer, Berlin, 1981, 216-239.

[7] R.S.Palais, The classification of $G$-spaces, Memoirs AMS, 36, 1960.

[8] L.S. Pontrjagin, Topological groups, Princeton Univ. Press, 1939.

[9] D.G.Quillen, Homotopical algebra, Lecture Notes in Math. 43, Springer, 1967.

A. Bykov

Benemérita Universidad Autónoma de Puebla

Facultad de Ciencias Fisico Matematicas

Av. San Claudio y Rio Verde

Ciudad Universitaria, Colonia San Manuel

CP 72570, Puebla, Pue.

México

E-mail: abykov@fcfm.buap.mx

M. Texis

Benemérita Universidad Autónoma de Puebla

Av. San Claudio y Rio Verde

Ciudad Universitaria, Colonia San Manuel

CP 72570, Puebla, Pue.

México

Received: 21.1.2004.

Revised: 2.3.2005. 